\title{
ANÁLISIS DE LA RELACIÓN ENTRE LA COMUNICACIÓN INTEGRADA DE MARKETING Y LA SATISFACCIÓN DEL CLIENTE. EL CASO MCDONALD'S.
}

\section{ANALYZING THE RELATIONSHIP BETWEEN INTEGRATED MARKETING COMMUNICATION AND CUSTOMER SATISFACTION. INSIGHTS FROM THE CASE OF MCDONALD'S.}

Asma Ahmed Laroussi (Universidad de Granada) ${ }^{1}$

\section{Resumen:}

La comunicación integrada de marketing (CIM) es un concepto que ha atraído gran interés y atención por parte de los académicos y de los profesionales del marketing. Sin embargo, la mayor parte de los estudios existentes se basa sobre la perspectiva organizativa, siendo la CIM percibida por el consumidor una de las lagunas de investigación más destacadas. Por tanto, este trabajo aborda la CIM desde la perspectiva del consumidor y se propone la consecución de un objetivo clave: determinar si la CIM contribuye a la obtención de una mayor satisfacción del cliente, que representa una variable crucial para el éxito de una empresa en el mercado actual. Para cumplir el objetivo propuesto, se llevó a cabo una encuesta online, de la que se obtuvo una muestra final de 252 clientes de la marca McDonald's. El análisis de los resultados permitió demostrar una relación positiva y significativa entre la coherencia del mensaje y la interactividad, dos dimensiones clave de la CIM, y la satisfacción del cliente. Finalmente, el trabajo propone una serie de implicaciones y futuras líneas de investigación.

Palabras Clave: Comunicación Integrada de Marketing, interactividad, coherencia del mensaje, satisfacción del cliente.

Códigos JEL: M31.

\begin{abstract}
:
The concept of Integrated Marketing Communication (IMC) is a concept that has attracted the marketing academics and practitioners' attention. Nevertheless, the great majority of existing studies is based on the organisational perspective and IMC in terms of consumers' perceptions is one of the main research gaps in marketing research. With these premises, the present work addresses IMC taking the customer's perspective and aims at determining whether IMC contributes to achieve a greater customer satisfaction, which is considered an essential variable for company success in today's market. To address this goal, an online survey was carried out among individuals who usually consume goods bearing the McDonald's trademark, resulting in a final sample of 252 individuals. The analysis of the results obtained led to demonstrate a significant positive relation between two key IMC dimensions, message consistency and interactivity, and
\end{abstract}

\footnotetext{
1 e.asmamed@go.ugr.es, Universidad de Granada.

Recibido: 4 de noviembre de 2020. Aceptado: 30 de noviembre de 2020.
} 
customer satisfaction. Finally, this paper draws several managerial implications and suggests future lines of research in this area.

Key Words: Integrated Marketing Communication, interactivity, message consistency, customer satisfaction.

JEL Codes: M31.

\section{INTRODUCCIÓN}

La Comunicación Integrada de Marketing surgió como concepto a finales de los años ochenta y, una década después, comenzó su investigación en el ámbito académico, dándose a conocer la primera definición de la CIM propuesta por Caywood et al. (1991) y, desde este punto de partida, se logró su crecimiento a lo largo de los últimos treinta años (Porcu et al., 2017).

La aparición de este concepto llevó a numerosos investigadores a debatir la validez de su fundamento. Por ejemplo, Cornelissen (2000) argumentó que la CIM representaba sólo una moda temporal sin un apoyo académico suficiente, afirmación que fue rebatida, más tarde, por Schultz y Kitchen (2000), quienes defendieron el concepto y enfatizaron el hecho de que, al tratarse de un concepto que se encontraba en un estado germinal, precisaba todavía de mayores esfuerzos por parte de los investigadores para el desarrollo de un marco teórico más sólido. Asimismo, en los años noventa muchos académicos se interesaron por el análisis de las facetas o dimensiones del concepto de CIM. Por ejemplo, los autores Duncan y Everett (1993) determinaron que la CIM era tanto un concepto como un proceso y, por tanto, era difícil llegar a una definición que reflejara la CIM en su totalidad.

Schultz y Kitchen (1997), por su parte, también afirmaron, en un estudio exploratorio de CIM sobre agencias de publicidad de EE. UU., que la literatura sobre CIM hasta ese momento se había centrado principalmente en aplicarla en lugar de entender sus principios y teorías básicas. Estos autores concluyeron que una conceptualización de la CIM más relevante y aceptable es crucial para promover el crecimiento y la práctica de este término en las organizaciones. En los últimos años, se considera que la comunicación integrada de marketing ha llegado a tener un valor estratégico para las organizaciones contemporáneas. Por ello, la implementación real de CIM por parte de las organizaciones hacia los clientes sigue siendo objeto de investigación, representando un enigma para los académicos (Šerić, 2018; Manoli y Hodgkinson, 2020).

Del mismo modo, un concepto que también ha generado un alto nivel de dedicación por parte de los académicos es la satisfacción del cliente, puesto que se trata de un pilar fundamental para las empresas. Este hecho ha implicado la realización de estudios exhaustivos en los que se han explorado métodos adecuados para medir la satisfacción del cliente y, sobre todo, estrategias para obtenerla (Ryu y Han, 2011). Por ejemplo, la satisfacción del cliente es lograda cuando se superan las expectativas del cliente ante el consumo de productos o servicios. Por otro lado, desde el enfoque basado en el rendimiento, ciertos autores han argumentado que la satisfacción del cliente necesita integrar respuestas afectivas además de las evaluaciones cognitivas durante el consumo (Oliver, 1993; De Rojas y Camarero, 2008). Por tanto, la necesidad de comprender la 
satisfacción desde un aspecto más emocional se ha enfatizado en la conexión con influencia cognitiva (Phillips y Baumgartner, 2002; Premkumar y Bhattacherjee, 2008; De Rojas y Camarero, 2008). En este sentido, dentro del concepto de marketing relacional, la relación entre la Comunicación Integrada de Marketing y la satisfacción del cliente se considera clave, ya que una mayor cercanía o intensidad de la relación entre una empresa y sus clientes puede llevar a una mayor satisfacción (Rather et al., 2019). Por tanto, este trabajo aborda la CIM desde la perspectiva del consumidor y se propone la consecución de un objetivo clave: determinar si la CIM contribuye a la obtención de una mayor satisfacción del cliente, que representa una variable crucial para el éxito de una empresa en el mercado actual.

\section{REVISIÓN DE LA LITERATURA}

\subsection{La Comunicación Integrada de Marketing y su papel en el sector de la restauración}

La mayor parte de la literatura sobre comunicación integrada de marketing (CIM) ha reconocido su importancia, considerándolo un componente estratégico esencial en el mundo empresarial (Duncan y Mulhern, 2004; Kitchen y Schultz, 2009; Kliatchko y Schultz, 2014; Porcu et al., 2016; Tafesse y Kitchen, 2017; Ahmad y Salleh, 2019; Albinsson et al., 2020).

Durante los últimos tiempos, autores como Ots y Nyilasi (2015) señalaron que la CIM seguía siendo todavía un importante objeto de investigación y que existen limitadas evidencias empíricas que demuestran los efectos que derivan de su implementación. En esta línea, estos autores señalaron que la CIM es un proceso comercial estratégico que involucra diferentes stakeholders para la comunicación de marca. Sin embargo, aún se desconocen las razones que justifican la dificultad de su implementación práctica.

Para ello, Ots y Nyilasi (2015) llevaron a cabo una investigación que proporcionaba una explicación novedosa para tales errores. De este estudio, los autores elaboraron cuatro modelos mentales básicos que, a su vez, revelaron cuatro aspectos fundamentales de la disfunción de implementación de CIM: falta de comunicación, excesiva departamentalización, pérdida de confianza y descontextualización. Según estos autores, la gestión de los modelos mentales de aquellos que llevan a cabo la CIM es esencial para hacer que este concepto sea un éxito en el ámbito del marketing y es una condición necesaria para que este ámbito de estudio pueda avanzar y contribuir a una implementación exitosa de la CIM.

Por otro lado, Moriarty y Schultz (2012) transmitieron la idea de que los académicos siempre han reconocido que el contexto organizacional de la implementación de la CIM es un área importante para la investigación empírica.

Desde su perspectiva, Peltier et al. (2003) destacaron la creciente importancia y el potencial de la naturaleza interactiva de los nuevos medios y su papel en la generación de interacción con los clientes, a través de la propuesta de una "CIM interactiva".

Por tanto, es posible concluir que el debate entre académicos y profesionales sobre la CIM sigue abierto, siendo la medición de los programas de CIM una de las cuestiones más difíciles de resolver desde los orígenes de este concepto. En esta línea, Cook (2004) apoya los argumentos de los autores que consideran que la CIM está en continua evolución y que se está expandiendo más allá del marketing.

Por su parte, con una visión más amplia, Kliatchko (2002) opinó que la CIM puede considerarse conceptualmente un término histórico, pero operativamente actual. Es decir, 
este autor considera que la CIM, en términos de concepto, está envuelta en dos principios fundamentales: (1) integración o coordinación en sí mismo y (2) la orientación al consumidor. Sin embargo, es operativamente actual porque los avances tecnológicos han hecho que sea más necesaria la integración y coordinación de los mensajes. Además, gracias a la tecnología, es posible para los profesionales del marketing poner en práctica la integración y la orientación al cliente.

Siguiendo con el debate acerca de la necesidad de una mayor investigación sobre el concepto, Kliatchko (2005) sugiere que la CIM se ha convertido en una tendencia irreversible entre académicos y profesionales de la industria. Por ello, este autor, en lugar de considerarlo como una revolución en el pensamiento de marketing, parte de que la CIM surgió como una evolución natural en la comunicación de marketing, provocada por cambios drásticos en al menos tres áreas principales: el mercado, los medios de comunicación y el consumidor. Por su parte, Schultz y Kitchen (2000) afirmaban que son cuatro los elementos que impulsan los cambios en el mercado actual, es decir, la práctica de las comunicaciones de marketing viene dada por la digitalización, la tecnología de la información, la propiedad intelectual y los sistemas de comunicación. Pese a estos conocimientos, estos autores apoyaban la idea de que era necesario conocer los antecedentes para seguir investigando.

Tal y como sugieren los autores anteriores, es necesario echar la vista atrás para analizar el origen de la CIM. En este sentido, según la primera definición de CIM, propuesta por Caywood et al. (1991) y adoptada por la American Association Advertising Agencies, este concepto representa la coordinación y coherencia del mensaje, el uso de una variedad de disciplinas de comunicación para trabajar en una sinergia basada en un plan exhaustivo.

Entre otras primeras definiciones de CIM más destacables, se encuentra la que facilitan Duncan y Caywood (1996), quienes definieron la CIM como:

'un concepto de planificación de comunicaciones de marketing que reconoce el valor de un plan integral que evalúa los roles estratégicos de una variedad de disciplinas de comunicación: publicidad general, marketing directo, promoción de ventas, y relaciones públicas. La combinación de estas disciplinas tiene la función de proporcionar claridad, coherencia y máximo impacto de comunicación'.

Si bien unos años antes, Nowak y Phelps (1994), a pesar de no proponer una definición explícita de CIM, optaron por tres “conceptualizaciones” que extrajeron de la literatura existente sobre la CIM: comunicaciones de marketing de una sola voz, comunicación integrada de marketing y comunicación coordinada de marketing.

Entre los autores que reconocieron que el radio de acción de la CIM va más allá del marketing se encuentran Schultz y Schultz (1998), quienes propusieron una nueva definición de CIM para captar el alcance actual y futuro de CIM tal y como lo han visto desarrollarse. Esta definición se basa en los estudios de CIM que se han realizado en el pasado, así como en las experiencias de las organizaciones que han implementado su enfoque. En este sentido, Schultz y Schultz (1998) definieron la CIM como:

"La CIM es un proceso comercial estratégico utilizado para planificar, desarrollar, ejecutar y evaluar programas de comunicación de marca coordinados, medibles y persuasivos a lo largo del tiempo con consumidores, clientes, prospectos y otras audiencias internas y externas específicas $y$ relevantes.” 
Por otro lado, mientras que Kliatchko (2008) afirmaba que las primeras investigaciones del desarrollo de la CIM se centraban en las definiciones de los conceptos, los trabajos más recientes tales como Tafesse y Kitchen (2016) y Porcu et al. (2017) aseguran que lo más relevante es la medida.

En concreto, Kliatchko (2008, 2009) considera la CIM como la evolución de la armonización táctica y una integración estratégica comercial expresándola como:

"un proceso empresarial impulsado por la audiencia para gestionar estratégicamente los stakeholders, el contenido, los canales y los resultados de los programas de comunicación de marca”.

Además, Kliatchko (2008) expuso que los gestores encargados de comunicación de marketing tenían que lidiar con problemas no resueltos relacionados con la creación de contenido en los medios. Estos problemas están relacionados con la idoneidad, conveniencia, adecuación con una coherencia de contenido creado por el consumidor con la visión de marca y las consideraciones estratégicas, así como suplantación de identidad, derechos de autor y fiabilidad del contenido. En definitiva, son éstas algunas de las preocupaciones más importantes que los gerentes de CIM deben tratar y enfrentar a la hora de exponer y utilizar estos medios.

Finalmente, para culminar la recopilación de definiciones que tratan de describir la CIM, se estudia cómo Porcu et al. (2017) conceptualizaron la CIM, basándose en una extensa revisión de definiciones y modelos teóricos que los llevó a definir la CIM como:

"El proceso interactivo centrado en las partes interesadas de planificación interfuncional y la alineación de procesos organizacionales, analíticos y de comunicación que permiten la posibilidad de diálogo transmitiendo mensajes coherentes y transparentes a través de todos los medios para fomentar a largo plazo relaciones rentables que crean valor."

Según Porcu et al. (2017) la CIM es un concepto multidimensional que recoge cuatro dimensiones: la coherencia del mensaje, la interactividad, el enfoque estratégico orientado hacia los stakeholders y la alineación organizacional. Este trabajo se centra en las dos primeras dimensiones, pues son las que el consumidor puede evaluar.

Estos autores consideran que "la coherencia del mensaje junto con las estrategias posicionamiento, la coherencia en los componentes visuales de la comunicación y la coherencia entre los mensajes de producto y servicio son los tres elementos principales capturados por esta dimensión".

Esta dimensión hace referencia al primer nivel de CIM, propuesto por Schultz y Schultz (1998), es decir, a la coordinación de todos los elementos de las comunicaciones de marketing para lograr sinergia y coherencia.

Por otro lado, la dimensión de interactividad fue definida por Porcu et al. (2017) como "el elemento sine qua non para establecer una comunicación simétrica bidireccional, una condición esencial para el diálogo entre la organización / marca y todos los interesados”.

Porcu et al. (2017) indican que tal y como se presenta la situación actual, es esencial para las organizaciones promover adecuadamente sus marcas entre los usuarios finales no sólo para eclipsar a los competidores, sino también para competir a largo plazo. Esta acción, de acuerdo con Selvakumar (2014), se logra con la promoción de la marca ya que aumenta la conciencia de los productos y servicios y, finalmente, aumenta sus ventas, lo que genera grandes ganancias e ingresos para la organización. Además, la CIM impulsa el logro de un rendimiento superior a una empresa, en particular para aquellas empresas que emplean la inteligencia de mercado (acciones de competencia y cambios en las 
preferencias del cliente) y sabe adaptarse a los cambios en el entorno (la aparición de nuevas tecnologías) (Butkouskaya et al., 2020).

Los expertos de marketing dedican una gran atención hacia los clientes, ya que éstos son los que influyen a la hora de tomar decisiones (Mulhern, 2009 y Kliatchko, 2009). En este sentido, la comunicación es un vehículo que permite a las empresas acercarse a sus clientes y convertirlos en potenciales, transmitiendo mensajes que informen adecuadamente sobre el producto o servicio ofrecido (Camilleri, 2018). De este modo, las compañías también conocen y descubren la evaluación de ciertos aspectos de los productos o servicios desde el punto de vista de sus clientes. La comunicación integrada es una herramienta enfocada a la transmisión de mensajes coherentes y persuasivos coordinados mediante canales que maximizan su impacto (Šerić, 2018; Camilleri, 2018) y el efecto en términos de satisfacción del cliente resulta ser de crucial importancia.

Para obtener ese valor de marca, Mora (2013) sugiere que la CIM cuenta con herramientas como la publicidad, las relaciones públicas, los medios masivos y el marketing digital, que dan a la empresa un impulso derivado de la coordinación, logrando así el éxito de una empresa. Este autor investigó sobre cómo los establecimientos de ocio aplican las herramientas de la CIM. Para ello, midió la utilidad de la CIM en restaurantes y concluyó que los establecimientos que aplican más de cuatro herramientas de CIM son lugares con un gran rendimiento y posicionamiento, así como diferenciación. De este modo, se puede analizar la empresa objeto a investigar, McDonald's como establecimiento de restauración dentro de la categoría de la restauración y el ocio, aplicando dichas herramientas de la CIM. Al mismo tiempo, las herramientas de CIM van de la mano con la tecnología. Por ello, las empresas, sobre todo las que se dedican a actividades de ocio, deben aprovechar las oportunidades facilitadas por los grandes avances tecnológicos. En este sentido, las TIC se han convertido en las aliadas de las empresas ya que éstas permiten conocer mejor a los clientes, de modo que influyen significativamente en sus estrategias de marketing y comunicación (Šerić y Gil-Saura, 2012).

Para ello, deben llevar a cabo adaptaciones a los avances e innovaciones tecnológicas para poder anticiparse y enfrentarse a las invariabilidades que surgen en el mercado. Y esta es la razón por la que autores como Šerić y Saura (2012) defienden la incorporación de la CIM en las estrategias de marketing, siempre que la comunicación sea eficazmente integrada y transmita un mensaje claro y coherente por vía de las herramientas de las que este proceso dispone.

También es muy importante que los consumidores perciban la coherencia del mensaje. Šerić y Saura (2012) demostraron, en su estudio, que la percepción de los huéspedes tiene la capacidad de influir significativamente en la creación del valor de marca, sobre todo en la imagen, la calidad percibida y la lealtad. El mensaje que deben transmitir las empresas debe ser claro y coherente, es decir, los mensajes dirigidos a los clientes no tienen que ser totalmente iguales, sino que se deben complementar y no contradecirse (Šerić y Saura, 2012 y Torp, 2009).

Por otro lado, Kim y Kim (2005) examinaron las dimensiones subyacentes del valor de marca y cómo afecta el desempeño de las empresas en la industria de la hospitalidad, en particular, hoteles de lujo y cadenas de restaurantes. A raíz de estos objetivos, estos autores determinaron, siguiendo los resultados de su estudio empírico, que la lealtad a la marca, la calidad percibida y la imagen de marca son componentes importantes del valor de marca basado en el cliente. Se encontró una relación positiva entre los componentes del valor de marca basado en el cliente y el desempeño de las empresas en hoteles de lujo y cadenas de restaurantes. A su vez, ese valor de marca que se obtiene se debe atribuir a 
la aplicación de la CIM, sobre todo, en las dos dimensiones estudiadas pues son las que mejor definen la perspectiva del cliente ya que las puede evaluar éste mismo.

Una vez revisada la literatura del concepto de CIM, es interesante revisarla también desde un enfoque más práctico, aplicado a la realidad de una actividad empresarial. En este caso, se va a analizar la CIM dentro del sector de la hostelería, concretamente en el servicio de comida rápida. Y, para ello, se selecciona como restaurante objeto de investigación a la cadena de restaurantes McDonald's.

Partiendo de que McDonald's es considerada un fenómeno y compañía legendaria, que es conocida por la mayoría de las personas alrededor del mundo (Davydova, 2016), es necesario tener en cuenta su misión desde el principio, es decir, esta empresa pretende hacer que la marca McDonald's sea global y gane cierta ubicuidad.

Para ello, desarrolla la estrategia de su CIM a un nivel considerable de tal manera que los públicos siempre aprecian la presencia de la marca en la televisión, vallas publicitarias, radio, muestra de productos en películas, patrocinio mediante embajadores, además de promover programas de Responsabilidad Social Corporativa (Davydova, 2016).

Vignali (2001) señaló que "la compañía cree que la mejor manera de diferenciarse de la multitud es satisfacer a todos los clientes y empleados todo el tiempo” y, además, puso énfasis en que McDonald's conoce su público objetivo exacto y sus necesidades, por lo tanto, la marca es capaz de dar forma a la estrategia de comunicación respondiendo a las necesidades de estos grupos específicos. Es decir, el público objetivo es diverso y cada uno visita el restaurante por una razón acorde a sus características; por ejemplo, un padre puede querer llevar a los niños para una HappyMeal, una persona de negocios puede pasar para adquirir comida rápida de almuerzo mientras que un adolescente puede ir con sus amigos además de aprovechar el servicio extra de Wi-Fi gratis.

Por su parte, Dessner (2013) destaca otro punto clave del caso de McDonald's Corporation. Este factor determinante corresponde a las actividades de relaciones públicas de la marca. Además, este autor describe las relaciones públicas de la industria de restaurantes como únicas al elegir el contenido que incluye en el mensaje.

\subsection{La satisfacción del cliente y su papel en el sector de la comida rápida}

La literatura existente considera que la satisfacción del cliente desempeña un papel muy importante en el ámbito empresarial. No obstante, existen numerosas perspectivas diferentes que abordan este concepto (Gallarza y Gil, 2006; Molina et al., 2014).

Por ejemplo, Wu y Liang (2009) interpretan la satisfacción del cliente como el valor de su experiencia, es decir, es la respuesta que evalúa la experiencia del consumidor.

Por su parte, Akbar y Parvez (2009) definen la satisfacción del cliente como la reacción que se produce ante una evaluación metódica que valora lo que se espera y lo que realmente se ha recibido. En la misma línea, Antón et al. (2007) consideran que la satisfacción del cliente se alcanza cuando se han cumplido o superado sus expectativas sobre el rendimiento y la calidad del producto o servicio. En este sentido, la satisfacción no se producirá si la percepción del rendimiento no coincide con la percepción esperada (Tjiptono y Diana, 2000).

La satisfacción del cliente se establece como el estado psicológico resultante de la diferencia entre los sentimientos previos del cliente y la experiencia definitiva de consumo (Rather et al., 2019). Esta definición coincide con la de otros autores que también entienden la satisfacción del cliente como el sentimiento de placer o decepción 
resultante de comparar la valoración final de un producto o servicio en relación con su expectativa inicial (Singh, 2006). Por su parte, Akbar y Parvez (2009) conciben, del mismo modo, la satisfacción del cliente como una respuesta provocada ante el estímulo de la evaluación que determina la diferencia entre lo que se espera y lo que se recibe.

Por este motivo, Gronroos (2007), Ryu y Kim (2009) y Rather y Sharma (2018) coinciden en que es un determinante del retorno de un cliente como efecto de la satisfacción. Wallace y Kirk-Brown (2004) confirman este hecho, asegurando que la satisfacción es un medio importante para lograr la lealtad. Es decir, la satisfacción provoca la repetición del comportamiento y la recomendación a través de comentarios positivos acerca de la experiencia de consumo (Gibson, 2005), puesto que este autor en un estudio de la industria de la hospitalidad, descubrió que los clientes satisfechos se convierten en repetidores de compra de productos o servicios y proporcionan a sus familiares y conocidos recomendaciones y opiniones positivas sobre sus experiencias.

Desde un punto de vista más concreto, Andaleeb y Conway (2006), a partir del desarrollo de sus estudios, estudiaron el papel de la satisfacción del cliente en el sector de la restauración, dando lugar a tres factores determinantes: calidad de servicio, calidad de producto y precio. Para estos autores, la calidad del producto se evalúa teniendo en cuenta dos aspectos que son la calidad de la comida y el lugar donde se sirve. Por esta razón, incluyen en su modelo la influencia positiva del diseño y la apariencia del restaurante en la satisfacción del cliente. Después de aplicar el modelo, los resultados demostraron que esta variable no era significativa para el modelo, por lo cual concluyeron que no es un aspecto fundamental para lograr la satisfacción del cliente. Sin embargo, hay autores que se han interesado en investigar más acerca del modo en que el ambiente de un lugar donde se presta un servicio influye en los sentimientos de las personas. Andaleeb y Conway (2006) incluyen, además, el precio dentro de su modelo. Para ellos, el precio puede atraer o repeler a los clientes, además de que influye en las expectativas del cliente, es decir, si el precio es alto se espera una alta calidad y si el precio es bajo cuestiona la habilidad del restaurante para entregar el producto y la calidad del servicio.

Por otro lado, Wilson (2002) investigó métodos para medir la satisfacción del cliente y comprar el nivel de la misma. Para ello, propuso el método de preguntas y recopilación de datos para medir la satisfacción. Intan et al. (2015), aplicando un tratamiento similar, analizaron la satisfacción del cliente midiendo el efecto que ejercía sobre la lealtad, culminando con el hallazgo de que la satisfacción que se obtiene por la calidad del servicio influye positivamente en la lealtad del cliente.

La calidad del servicio y del producto y el precio, así como el diseño del establecimiento y la atmósfera de éste son elementos que determinan los sentimientos encontrados de los clientes (Andaleeb y Conway, 2006; Baker et al., 2002; Minor et al., 2004).

Como se ha apreciado en la literatura, la satisfacción se puede medir por medio de los elementos que componen el servicio. En este sentido, cabe destacar que los restaurantes disponen de elementos propios que los caracterizan. En un restaurante todos los detalles influyen en la percepción del cliente acerca del servicio. Por ejemplo, el entorno es un elemento importante que todo restaurante debe cuidar pues da lugar a ciertas interacciones del cliente con los elementos in/tangibles y tangibles que forman la atmósfera del lugar y las señales ambientales en el mismo (Baker et al., 2002; Keng et al., 2007).

Por otro lado, el trato de los empleados es un gran aliado para lograr la satisfacción del cliente a través de una alta calidad de atención al cliente, organización del personal y 
su profesionalidad, desarrollando, de este modo, una relación interactiva entre clientes y empleados pues será una clave para una evaluación positiva del servicio por parte de los clientes (Andaleeb y Conway, 2006; Wu y Liang, 2009).

Por su parte, Wu y Liang (2009) destacaba la eficiencia temporal pues se trata de un elemento significativo para la satisfacción del cliente, sobre todo, para la valoración de restaurantes de comida rápida.

Por tanto, se puede concluir con que todos los elementos construyen el concepto de calidad de servicio por el cliente gastronómico. Por esta razón, se ha convertido en un tema estratégico para las empresas.

\subsection{La relación entre la CIM y la satisfacción del cliente}

Durante las últimas décadas, la Comunicación Integrada de Marketing se ha analizado desde una perspectiva gerencial, pasando por alto la posición del cliente y la influencia en su satisfacción, lo que supone una vía de investigación pendiente de abordar. Por ello, en los últimos años, numerosos académicos se dedicaron a analizar el concepto desde una visión multidimensional lo que ha implicado que las dimensiones de la Comunicación Integrada de Marketing supongan una ventaja competitiva para las empresas.

Existen hallazgos que explican y demuestran la relación entre los patrones de interacción y comunicación de marketing entre compañía y clientes, testeando sus expectativas lo que permite detecta su nivel de satisfacción (Park et al., 2018; Chamidah et al.2020).

Por ejemplo, en el contexto económico actual, el consumidor medio experimenta una multitud de estímulos como resultado de la aplicación de la CIM por parte de las diferentes empresas, las compañías exponen sus marcas a través de la comunicación creando una imagen unitaria de su actividad con el fin de atraer y retener clientes (Duralia, 2018; Perreault, 2018).

De este modo, el movimiento de la economía implica una feroz competencia entre los diferentes empresarios que luchan por llamar la atención de clientes ofreciendo una diversidad continua para satisfacer las necesidades heterogéneas (Duralia, 2018; Bernyte, 2018). Por este motivo, la comunicación integrada de marketing actúa de puente para que las grandes empresas obtengan una actitud favorable de los consumidores hacia su producto o servicio (Oluwafemi y Adebiyi, 2018). Oluwafemi y Adebiyi (2018) estudiaron la relación de la satisfacción y lealtad del cliente y la CIM en el sector de las telecomunicaciones concluyendo, finalmente, que existe una importante influencia de las dimensiones de la CIM en la lealtad del cliente, generando también competencia entre las empresas de esta industria.

Por otro lado, Jung (2016), en su estudio sobre las relaciones influyentes entre menús de restaurantes, dimensiones de la CIM y satisfacción del cliente afirmó que la comunicación con el cliente ha supuesto una influencia significativa en su satisfacción, estableciéndola como una estrategia de marketing. Por su parte, Butkouskaya et al. (2020), en su reciente investigación, afirmaron que la CIM afecta positivamente a la satisfacción del cliente que, a su vez, impacta positivamente en WOM y en la intención de recompra. La búsqueda de canales y programas persuasivos tienen el fin de construir una comunicación basada en mensajes coherentes con clientes y otras partes interesadas, implicando, de este modo, una interactividad entre los mismos.

La aplicación de la CIM tiene el fin de transmitir mensajes coherentes en los diversos canales, lo que repercute positivamente en la evaluación del cliente sobre la 
comunicación y, por tanto, influye directamente en su nivel de satisfacción (Low, 2000; Vorhies et al., 2009; Danaher y Rossiter, 2011).

Por ejemplo, la información percibida de un mensaje publicitario debe ser igual a sus expectativas. De este modo, el cliente experimentará un nivel de satisfacción a la hora de evaluar el servicio, lo que influirá también en su decisión de recompra (Mihart, 2012). Por tanto, la positiva experiencia que genera una adecuada comunicación basada en mensajes coherentes y en el desarrollo de la interactividad debería mejorar la satisfacción del cliente (Reid, 2005; Šerić et al., 2014).

Por tanto, basándose en la revisión de literatura de la CIM como concepto multimensional, se seleccionan la coherencia del mensaje y la interactividad como las dimensiones más susceptibles de ser evaluadas por el cliente para comprobar si existe una relación positiva con su satisfacción, dando lugar a una nueva aplicación de la CIM dentro del sector de la restauración.

Para ello, se propone un modelo teórico en el que se analizan factores influyentes en la satisfacción (véase Figura 1) donde se va a medir la relación directa entre las dimensiones que forman la CIM y la satisfacción.

Siguiendo este criterio, se plantean las siguientes hipótesis:

$\mathrm{H}_{1}$ : La coherencia del mensaje influye positivamente en la satisfacción.

$\mathrm{H}_{2}$ : La interactividad influye positivamente en la satisfacción.

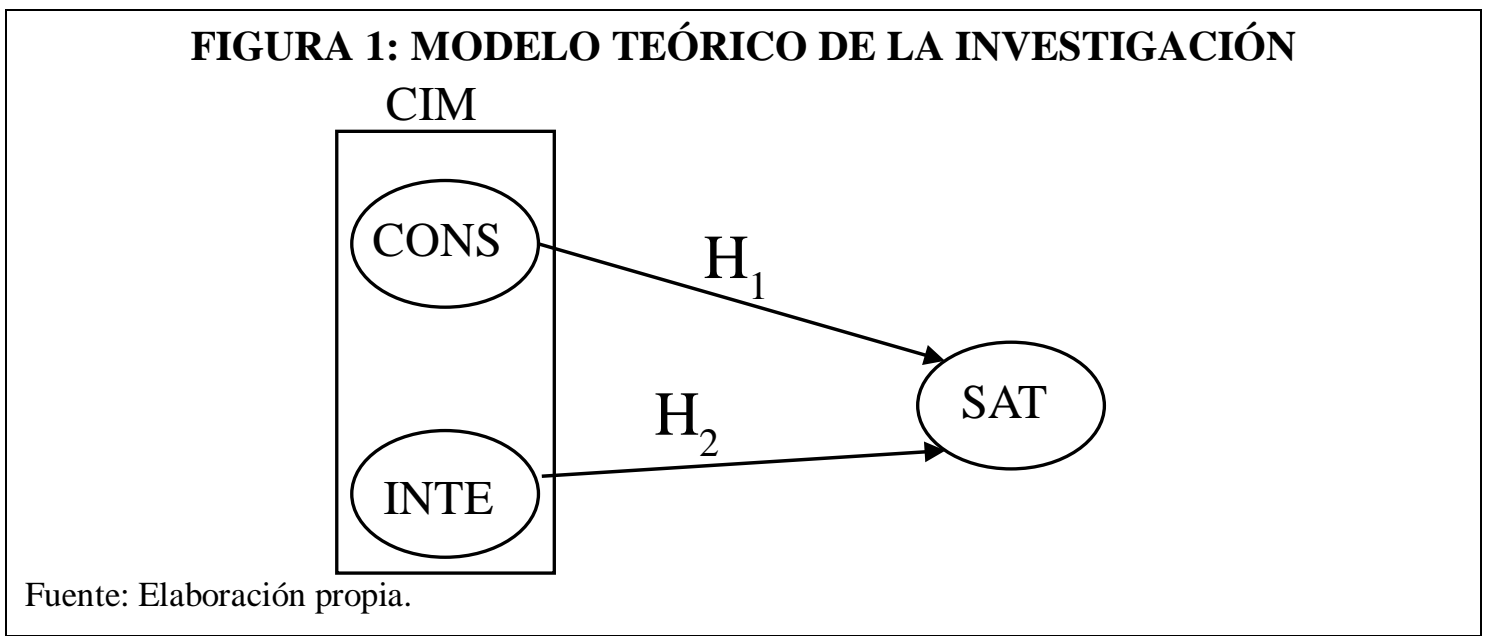

\section{METODOLOGÍA}

\subsection{Procedimientos para la recogida de datos}

La población objetivo de este estudio corresponde a consumidores de McDonald's. Los usuarios se han identificado según datos demográficos como la edad, el género, la situación laboral y otros datos relacionados con la visita al restaurante, como la compañía y la frecuencia. El tipo de muestreo es de bola de nieve y los datos se recogieron a través de una encuesta online en el mes de junio de 2018.Finalmente se obtuvo una muestra de 252 casos.

El cuestionario se elaboró y se alojó en la platafoma web Qualtrics accesible a través de un enlace (https://webcim.qualtrics.com/jfe/form/SV cObHqs30CsylaFD) que se adjuntó a una invitación distribuida a través de las redes sociales más destacadas: 
Whatsapp, Instagram, Facebook y Twitter. La tabla 1 presenta las características sociodemográficas de la muestra.

\begin{tabular}{|c|c|c|c|}
\hline \multicolumn{4}{|c|}{ TABLA 1: DESCRIPCIÓN DE LA MUESTRA } \\
\hline & & $\mathbf{n}$ & Porcentaje \\
\hline Género & $\begin{array}{l}\text { Hombre } \\
\text { Mujer }\end{array}$ & $\begin{array}{l}92 \\
160\end{array}$ & $\begin{array}{l}36,50 \% \\
63,50 \%\end{array}$ \\
\hline Edad & $\begin{array}{l}\text { Entre } 16 \text { y } 25 \\
\text { Entre } 26 \text { y } 35 \\
\text { Entre } 36 \text { y } 50 \\
\text { Más de } 51\end{array}$ & $\begin{array}{l}104 \\
107 \\
35 \\
6\end{array}$ & $\begin{array}{l}41,27 \% \\
42,46 \% \\
13,89 \% \\
2,38 \%\end{array}$ \\
\hline Situación laboral & $\begin{array}{l}\text { Estudiante } \\
\text { Trabajador por cuenta ajena } \\
\text { Trabajador autónomo } \\
\text { Pensionista } \\
\text { Desempleado } \\
\text { Otros }\end{array}$ & $\begin{array}{l}106 \\
67 \\
11 \\
2 \\
24 \\
42\end{array}$ & $\begin{array}{l}42,06 \% \\
26,59 \% \\
4,37 \% \\
0,79 \% \\
9,52 \% \\
16,67 \%\end{array}$ \\
\hline Compañía & $\begin{array}{l}\text { Solo } \\
\text { Con la familia } \\
\text { Con amigos } \\
\text { Compañeros de trabajo } \\
\text { Con mi pareja }\end{array}$ & $\begin{array}{l}25 \\
86 \\
117 \\
0 \\
24\end{array}$ & $\begin{array}{l}9,92 \% \\
34,13 \% \\
46,43 \% \\
0,00 \% \\
9,52 \%\end{array}$ \\
\hline Frecuencia & $\begin{array}{l}\text { Un par de veces al mes o menos } \\
\text { Tres veces al mes } \\
\text { Cuatro veces al mes } \\
\text { Más de cuatro veces al mes }\end{array}$ & $\begin{array}{l}195 \\
30 \\
12 \\
15\end{array}$ & $\begin{array}{l}77,38 \% \\
11,90 \% \\
4,76 \% \\
5,96 \%\end{array}$ \\
\hline
\end{tabular}

\subsection{Escalas de medida de las variables incluidas en el estudio}

El diseño del cuestionario se ha basado en escalas multi-ítems tipo Likert de 7 puntos (de 1, totalmente en desacuerdo, a 7, totalmente de acuerdo). No hubo que eliminar respuestas, puesto que la herramienta utilizada solo admitía los cuestionarios con respuestas a todos los ítems establecidos y que fueran realizados en al menos 10 minutos de tiempo.

El cuestionario está compuesto por dos partes. La primera parte identifica los datos sociodemográficos de los participantes: género, edad, situación laboral, el tipo de compañía con quien suele acudir al restaurante y la frecuencia de visita. La segunda parte corresponde con la medición de la CIM a través de la coherencia del mensaje y la interactividad y de la satisfacción. Para ello, se adaptó la escala de Porcu et al. (2017), con el fin de que pudiese reflejar la CIM de McDonald's desde el punto de vista de los clientes, mientras que la satisfacción del cliente fue medida mediante la escala de Wu y Liang (2009) formada por tres ítems (véase Tabla 2). 


\section{TABLA 2: ESCALAS DE MEDIDA DE LA COMUNICACIÓN INTEGRADA DE MARKETING Y DE LA SATISFACCIÓN DEL CLIENTE}

\begin{tabular}{|c|c|}
\hline Constructos & Ítems \\
\hline \multirow{4}{*}{$\begin{array}{l}\text { Coherencia } \\
\text { del mensaje }\end{array}$} & $\begin{array}{l}\text { CONS1: McDonald’s coordina cuidadosamente todos los mensajes } \\
\text { originados por todos los departamentos y funciones con el objetivo de } \\
\text { mantener la consistencia de su posicionamiento estratégico. }\end{array}$ \\
\hline & $\begin{array}{l}\text { CONS2: McDonald's mantiene consistencia en todos los componentes } \\
\text { visuales de la comunicación. }\end{array}$ \\
\hline & $\begin{array}{l}\text { CONS3: McDonald's revisa periódicamente todos sus mensajes } \\
\text { planificados para determinar su nivel de consistencia de posicionamiento } \\
\text { estratégico. }\end{array}$ \\
\hline & $\begin{array}{l}\text { CONS4: En McDonald's, es primordial mantener la coherencia entre los } \\
\text { mensajes del producto, que se infieren y comprenden todo lo que está } \\
\text { incrustado en los mensajes de productos y servicios de la organización, } \\
\text { derivados de la experiencia de tratar con la organización, su personal, } \\
\text { agentes y productos. }\end{array}$ \\
\hline \multirow{7}{*}{ Interactividad } & $\begin{array}{l}\text { INTE1: McDonald's promueve la creación de programas especiales para } \\
\text { facilitar las consultas y quejas de las partes interesadas sobre nuestras } \\
\text { marcas, productos y la propia empresa. }\end{array}$ \\
\hline & $\begin{array}{l}\text { INTE2: McDonald's reúne información de las partes interesadas que se } \\
\text { recopila o genera a través de diferentes fuentes de todas las divisiones o } \\
\text { departamentos en una base de datos unificada que está configurada para } \\
\text { ser útil y de fácil acceso para todos los niveles de la organización. }\end{array}$ \\
\hline & $\begin{array}{l}\text { INTE3: En McDonald’s, es crucial para la organización en su conjunto y } \\
\text { para todos sus recursos humanos tener una actitud receptiva hacia los } \\
\text { mensajes recibidos de sus partes interesadas. }\end{array}$ \\
\hline & $\begin{array}{l}\text { INTE4: En McDonald's, el uso estratégico de las TIC mejora la velocidad } \\
\text { de respuesta de la organización como un todo. }\end{array}$ \\
\hline & $\begin{array}{l}\text { INTE5: En McDonald's, escuchar activamente los mensajes generados por } \\
\text { las partes interesadas, por ejemplo, mediante el boca-oído (WOM y e- } \\
\text { WOM) es de vital importancia para establecer sus estrategias de } \\
\text { comunicación. }\end{array}$ \\
\hline & $\begin{array}{l}\text { INTE6: McDonald’s considera que la relación entre la empresa y sus } \\
\text { partes interesadas debe ser recíproca para establecer un diálogo basado en } \\
\text { la confianza y en curso. }\end{array}$ \\
\hline & $\begin{array}{l}\text { INTE7: McDonald’s implementa de forma proactiva las redes sociales } \\
\text { escuchando las conversaciones existentes para promover un diálogo con } \\
\text { sus grupos de interés. }\end{array}$ \\
\hline \multirow{3}{*}{$\begin{array}{c}\text { Satisfacción } \\
\text { del cliente }\end{array}$} & SAT1: Estoy satisfecho con el personal. \\
\hline & SAT2: Estoy satisfecho con la calidad del servicio. \\
\hline & SAT3: Estoy satisfecho en general \\
\hline
\end{tabular}




\section{RESULTADOS}

\subsection{Análisis de las propiedades psicométricas de las escalas}

Debido a que el tamaño de muestra obtenido (252) es superior a los 100 casos mínimos para considerarse representativo de la población (Churchill, 1979), se ha decidido determinar la validez y fiabilidad de las escalas por medio de un Análisis Factorial Confirmatorio (AFC) utilizando el software Lisrel 9.2., así como la estimación del modelo y el contraste de las hipótesis aplicando la modelización de ecuaciones estructurales.

En primer lugar, se procede a examinar si las escalas utilizadas tienen propiedades psicométricas adecuadas, es decir, determinar la fiabilidad y la validez que permiten medir los respectivos constructos. En la Tabla 3, se muestran los datos resultantes del AFC para realizar la comprobación de la validez y consistencia interna de las escalas empleadas en el cuestionario. Para ello, se ha empleado el software especializado en ecuaciones estructurales basadas en matrices de varianza-covarianza, mediante el procedimiento de estimación ML Robusto (Satorra y Bentler, 1988) que permite obtener las estimaciones pertinentes de los errores estándar con el fin de poder ajustar el valor de la Chi-cuadrado, en caso de que no sigan una distribución normal.

Según los datos obtenidos y el método aplicado, se debería rechazar el modelo puesto que presenta un valor del Chi-cuadrado de 180,81 y p-valor de 0,00 , quedando, de este modo, dentro de la zona de rechazo. No obstante, los índices de ajuste global del modelo, se puede observar que el Root Mean Square Error of Aproximation (RMSEA) presenta un valor de 0,076, por debajo de 0,08 (Hair et al. 2010; Del Barrio-García y Luque, 2012; Bagozzi y Yi, 2012) y los valores del CFI (0,99); IFI $(0,99)$, RFI $(0,98)$, NFI $(0.98)$ y NNFI $(0,98)$ son próximos a 1 . Por tanto, se concluye que el ajuste del modelo no se rechaza.

Según Hair et al. (2019), es imprescindible cumplir una serie de condiciones para confirmar la validez convergente de las escalas. Para ello, se comprobó la fiabilidad individual $\left(\mathrm{R}^{2}\right)$ de cada uno de los ítems. La fiabilidad se define como el nivel en que las medidas no contienen error, obteniendo, de este modo, resultados coherentes (Peter, 1979) por lo que el principal objetivo es eliminar aquellos que presenten un valor inferior a 0,50. En segundo lugar, se determina la fiabilidad compuesta (FC) del constructo que se obtiene mediante el cociente del sumatorio de las cargas estandarizadas entre la suma de éstas y el sumatorio de errores de medida (Porcu, 2014).

Basando la conclusión en dicho criterio, en la Tabla 3, se presentan los ítems que superaron el criterio de la fiabilidad individual. Todos ellos ofrecen un $\mathrm{R}^{2}>0,50$ por lo que todas las cargas son significativas ( $\mathrm{t}>1,96)$ (Bagozzi y Yi, 1988), lo que indica que se ha obtenido el mínimo necesario para tener en común con la variable subyacente a la que está unida (Porcu, 2014). En lo que respecta a la Fiabilidad Compuesta, todos los ítems presentan valores superiores al valor mínimo aceptado, 0,70 (Porcu, 2014; Hair et al. 2010; Del Barrio y Luque, 2012; Fornell y Larcker, 1981).

Por otro lado, analizando la Varianza Extraída, se reflejan valores superiores al mínimo de aceptabilidad, es decir, 0,50 (Porcu, 2014; Hair et al. 2010; Del Barrio y Luque, 2012; Fornell y Larcker, 1981). Además, cabe destacar que los coeficientes resultaron significativos ( $\mathrm{t}>1,96)$ (Bagozzi y Yi, 1988) (véase Tabla 3). 
TABLA 3: RESULTADOS DE LA VALIDACIÓN DE LAS ESCALAS DE MEDIDA

\begin{tabular}{|c|c|c|c|c|c|}
\hline Constructo & $\begin{array}{c}\text { Ítems } \\
\text { (etiquetas) }\end{array}$ & $\begin{array}{c}\text { Cargas estandarizadas } \\
\text { (valor t) }\end{array}$ & $\mathrm{R}^{2}$ & FC & $\mathrm{VE}$ \\
\hline \multirow{4}{*}{$\begin{array}{l}\text { Coherencia } \\
\text { del mensaje }\end{array}$} & CONS1 & $0,87(*)$ & 0,76 & \multirow{4}{*}{0,91} & \multirow{4}{*}{0,73} \\
\hline & CONS2 & 0,83 (15.97) & 0,69 & & \\
\hline & CONS3 & $0,83(15.82)$ & 0,68 & & \\
\hline & CONS4 & $0,88(17.40)$ & 0,77 & & \\
\hline \multirow{7}{*}{ Interactividad } & INTE1 & $0,74(*)$ & 0,55 & \multirow{7}{*}{0,94} & \multirow{7}{*}{0,69} \\
\hline & INTE2 & $0,83(15.87)$ & 0,68 & & \\
\hline & INTE3 & 0,88 (17.65) & 0,78 & & \\
\hline & INTE4 & $0,83(15.98)$ & 0,69 & & \\
\hline & INTE5 & 0,84 (16.39) & 0,71 & & \\
\hline & INTE6 & $0,84(16.20)$ & 0,70 & & \\
\hline & INTE7 & $0,86(16.99)$ & 0,74 & & \\
\hline \multirow{3}{*}{$\begin{array}{c}\text { Satisfacción } \\
\text { del cliente }\end{array}$} & SAT1 & $0,88(*)$ & 0,78 & \multirow{3}{*}{0,93} & \multirow{3}{*}{0,82} \\
\hline & SAT2 & 0,94 (19.67) & 0,89 & & \\
\hline & SAT3 & 0,89 (17.65) & 0,78 & & \\
\hline
\end{tabular}

Chi-cuadrado:180,81; gl: 74; p-valor: 0,0000; RMSEA: 0,076; NFI: 0.98; NNFI: 0,98; CFI: 0,99; IFI: 0,99; RFI: 0,98

Fuente: Elaboración propia.

La validez discriminante de las escalas se ha examinado aplicando el criterio de Fornell y Larcker (1981) y los resultados indican que la raíz cuadrada de la varianza extraída es, en todos los casos, superior a la correlación entre constructos. De acuerdo con Porcu (2014), la matriz de validez discriminante representa el nivel en que un constructo es fundamentalmente diferente de los demás (Hair et al., 2010). Por tanto, con estos resultados se demuestra que las escalas cuentan con validez discriminante.

\subsection{Estimación del modelo planteado y contraste de hipótesis}

La investigación actual ha proporcionado una nueva aplicación de la CIM, estableciendo una relación entre dos de sus dimensiones y la satisfacción del cliente, lo que implica un nuevo modo de entender y aplicar la CIM desde la perspectiva del cliente dentro del sector de la hostelería.

Tras la validación de las escalas, se procede con el contraste de las hipótesis a través de la estimación del modelo.

Los resultados obtenidos (Figura 2) indican que existe una relación positiva y significativa entre la coherencia del mensaje y la satisfacción del cliente $\left(\beta_{\text {cons } \rightarrow \text { sat }}=.66\right.$; $p<$.05), llevando a no rechazar la $\mathrm{H}_{1}$. Del mismo modo, la relación entre interactividad y satisfacción del cliente resultó ser positiva y significativa $\left(\beta_{\text {inte } \rightarrow \text { sat }}=.70 ; p<.05\right)$. Este resultado, permite concluir que la $\mathrm{H}_{2}$ no se rechaza. En resumen, los resultados permiten 
demostrar la relación positiva entre ambas dimensiones de la CIM, coherencia del mensaje e interactividad, y la satisfacción del cliente.

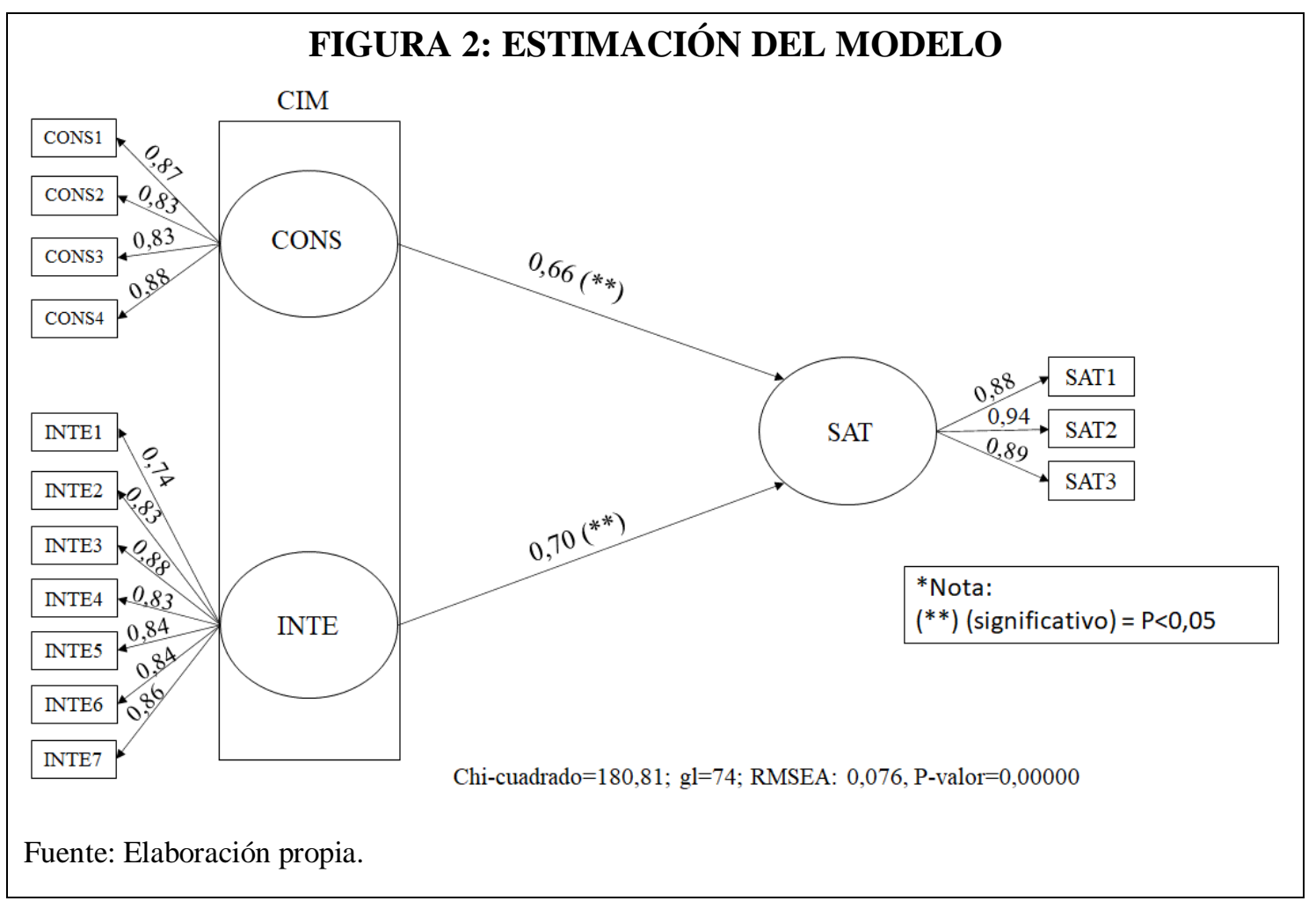

\section{CONCLUSIONES, IMPLICACIONES, LIMITACIONES Y FUTURAS LÍNEAS DE INVESTIGACIÓN}

Esta investigación ha tenido como fin abordar la perspectiva del cliente en su percepción de la CIM y su relación con la satisfacción. Para ello, se ha revisado la literatura de estos conceptos, destacando, en el caso de CIM, aquellas dimensiones que los clientes pueden valorar desde su posición externa. Los resultados sugieren que existe una fuerte relación positiva entre la coherencia del mensaje y la satisfacción y entre la interactividad y la satisfacción. En este sentido, se demuestra un mayor impacto en la satisfacción cuando los mensajes son coherentes y hay una mayor interactividad.

Este trabajo permite resaltar la gran importancia que tiene el buen uso de las herramientas de CIM por parte de la empresa ya que, desde el punto de vista del consumidor, el modo más óptimo de poner en marcha la CIM es a través de mensajes coherentes, la transmisión de una buena imagen y la interactividad con sus clientes. De esta forma, la empresa puede atender a las necesidades, inconformidades y sugerencias de sus clientes, respondiendo a ellas con eficiencia y eficacia.

En concreto, se recomienda a los directivos en el sector de la comida rápida, y en el caso específico de McDonald's, apostar por las herramientas de CIM que permitan mejorar la coherencia y la interactividad, orientando su misión a transmitir a sus clientes la imagen adaptada e interactuar con ellos para seguir obteniendo un feedback, que le permita conocer mejor y acertar a la hora de implantar nuevas estrategias. De este modo, tal y como se han mostrado en los resultados, la implantación de la CIM daría lugar a un 
mayor grado de satisfacción de sus clientes a través de mensajes coherentes y una mayor interacción y diálogo.

Los resultados de este trabajo representan un buen punto de partida para los gerentes de marketing que quieran adecuar sus estrategias llevadas a cabo en sus campañas de comunicación, adaptándose al país en el que estén o se quieran implantar. Por ello, se sugiere como futura línea de investigación analizar estas herramientas dentro del marco cultural de los diferentes países en los que opera McDonalds, llevando a cabo un análisis cross-cultural.

\section{BIBLIOGRAFIA}

Ahmad, A y Salleh, S. M, (2019). Brand Related Capabilities and the Process of Integrated Marketing Communications (IMC): A Resource Based View (RBV). Journal of Contemporary Research in Social Sciences, ${ }^{0} 1$, pp. 136-150.

Akbar, M. M, y Parvez, N, (2009). Impact of service quality, trust, and customer satisfaction on customer's loyalty. ABAC Journal, vol. 29, $\mathrm{n}^{\circ} 1$.

Albinsson, P. A, Burman, B, Shows, G. D, y Stoddard, J. E. (2020). Integrating and assessing student perceived sustainability literacy in an Integrated Marketing Communications course. Marketing Education Review, pp. 1-18.

Alcántara-Pilar, J. M., del Barrio-García, S., Crespo-Almendros, E., y Porcu, L. (2017). A review of psycho-vs. socio-linguistics theories: An application to marketing research”. In Advertising and Branding: Concepts, Methodologies, Tools, and Applications. IGI Global, pp. 1-30.

Andaleeb, S.S., y Conway, C. (2006). Customer satisfaction in the restaurant industry: an examination of the transaction-specific model. Journal of services marketing, $\mathrm{n}^{\mathrm{o}}$ 20, pp. 3-11.

Antón, C, Camarero, C, y Carrero, M. (2007). The mediating effect of satisfaction on consumers' switching intention. Psychology \& Marketing, vol. 24, n 6, pp. 511538.

Bagozzi, R. P, y Yi, Y, (1988). On the evaluation of structural equation models”. Journal of the academy of marketing science, vol. 16, $\mathrm{n}^{\circ} 1$, pp.74-94.

Baker, J, Parasuraman, A, Grewal, D, y Voss, G.B, (2002). The influence of multiple store environment cues on perceived merchandise value and patronage intentions". Journal of Marketing, vol. 66, n 2, pp. 120-141.

Bernyte, S, (2018). Sustainability marketing communications based on consumer values and principles. Regional Formation and Development Studies, vol. 26, $\mathrm{n}^{\mathrm{o}}$ 3, pp. 26-35.

Butkouskaya, V, Llonch-Andreu, J, y Alarcón-del-Amo, M. D. C. (2020). Inter-Country Customer-Perspective Analysis of Strategic Antecedents and Consequences for Post-Purchase Behaviour in Integrated Marketing Communications (IMC). Journal of International Consumer Marketing, pp. 1-16.

Butkouskaya, V, Llonch-Andreu, J, y Alarcón-del-Amo, M. D. C, (2020). Entrepreneurial Orientation (EO), Integrated Marketing Communications (IMC), and Performance in Small and Medium-Sized Enterprises (SMEs): Gender Gap and Inter-Country Context. Sustainability, vol. 12, nº 17, pp. 7159.

Butkouskaya, V, Llonch-Andreu, J, y Alarcón-Del-Amo, M.D.C (2019). Strategic antecedents and organisational consequences of IMC in different economy types. J. Mark. Commun.

Camilleri, M. A, (2018). Integrated marketing communications. In Travel marketing, tourism economics and the airline product, pp. 85-103. 
Caywood, C, Schultz, D.E, y Wang, P, (1991) Integrated marketing communications: A survey of national consumer goods advertisers: Research and Report”, Northwestern University, Evanston, IL.

Chamidah, N, Guntoro, B, y Sulastri, E, (2020). Marketing Communication and Synergy of Pentahelix Strategy on Satisfaction and Sustainable Tourism. The Journal of Asian Finance, Economics, and Business, 7(3), 177-190.

Churchill G. A, (1979). A paradigm for developing better measures of marketing constructs, Journal of Marketing Research, $\mathrm{n}^{\circ}$ 16, pp. 64-73.

Cook, W.A, (2004). IMC's Fuzzy picture: breakthrough or breakdown? Journal of Advertising Research, $\mathrm{n}^{\circ}$ 44, pp. 1-2.

Cornelissen, J, (2000). Corporate image: an audience centred model. Corporate Communications: An International Journal, vol. 5, nº 2, pp. 119-125.

Danaher, P. J, y Rossiter, J. R, (2011). Comparing perceptions of marketing communication channels. European Journal of Marketing, $n^{\circ}$ 45, pp. 6-42.

Davydova, A, (2016). Integrated Marketing Communications in Restaurant Chains: Identifying the Specifics of the Industry, (Doctoral dissertation, Empire State College).

Davydova, A, (2016). Integrated Marketing Communications in Restaurant Chains: Identifying the Specifics of the Industry (Doctoral dissertation, Empire State College).

De Rojas, C, y Camarero, C, (2008). Visitors' experience, mood and satisfaction in a heritage context: Evidence from an interpretation center. TourismManagement, vol. 29, n 3, pp. 525-537.

Del Barrio-García, S, y Luque-Martínez, T, (2012). Análisis de ecuaciones estructurales. Técnicas de análisis de datos en investigación de mercados, ed. T. Luque, 525À610. Barcelona: Pirámide.

Delener, N, y Neelankavil, J. P, (1990). Informational sources and media usage. Journal of Advertising Research, vol. 30, n 3, pp. 45-52.

Dessner, M, (2013). It's all the secret formula: Public Relations practices of the fast food industry (Master's thesis, Concordia University). Recuperado de: http://matthewdessner.com/text-based-work/

Duncan, T, y Caywood, C, (1996). The concept, process, and evolution of integrated marketing communication. Integrated communication: Synergy of persuasive voices, pp. 13-34.

Duncan, T.R, y Everett, S.E, (1993). Client perceptions of integrated marketing communications. Journal of Advertising Research, vol. 33, nº. 3, pp. 30-39.

Duncan, T.R, y Mulhern, F, (2004). A white paper on the status, scope and future of IMC (from IMC symposium sponsored by the imc programs at northwestern university and the university of denver). New York: McGraw-Hill: 9.

Duralia, O, (2018). Integrated marketing communication and its impact on consumer behavior. Studies in Business and Economics, vol. 13, n 2, pp. 92-102.

Engel, J. F, Blackwell, R. D, y Miniard, P. W, (1993). Alternative evaluation. Consumer Behaviour. Dryden Press, Fort Worth, Texas, pp. 533-556.

Fornell, C., y Larcker, D. F. (1981). Evaluating structural equation models with unobservable variables and measurement error". Journal of marketing research, pp. 39-50.

Gallarza, M. G, y Saura, I. G, (2006). Value dimensions, perceived value, satisfaction and loyalty: an investigation of university students' travel behaviour. Tourism management, vol. 27, $\mathrm{n}^{\mathrm{o}} 3$, pp. 437-452. 
Gibson, H, (2005). Towards an understanding of 'why sport tourists do what they do. Sport in Society, vol. 8, $\mathrm{n}^{\circ}$ 2, pp. 198-217.

Gilbert, G. R, y Veloutsou, C, (2006). A cross-industry comparison of customer satisfaction. Journal of Services Marketing, vol. 20, $\mathrm{n}^{0}$ 5, pp. 298-308.

Grönroos, C, (2007). Service management and marketing: customer management in service competition. John Wiley \& Sons.

Hair, J. F, Black, W. C., Babin, B. J, y Anderson, R. E, (2010). Multivariate Data Analysis, 7th edition, Pearson Prentice Hall: Upper Saddle River, New Jersey, Estados Unidos.

Hofstede, G, (1983). The cultural relativity of organizational practices and theories. Journal of international business studies, vol. 14, n 2, pp. 75-89.

Intan, M. A. R, Mohd, J. A. R, Bibi, N. M. Y, y Mohd, S.S, (2015). The impact of service quality and customer satisfaction on customer's loyalty: evidence from fast food restaurant of Malaysia. International Journal of Information, Business and Management, vol. 7, $\mathrm{n}^{\circ}$ 4, pp. 201-236.

Jung, J. W, (2016). The influence of menu choices and IMC activities on the customer satisfaction and the revisit intention at Italian restaurants. Culinary science and hospitality research, vol. 22, $\mathrm{n}^{\circ}$ 8, pp. 53-66.

Keng, C. J, Huang, T. L, Zheng, L. J, y Hsu, M. K, (2007). Modeling service encounters and customer experiential value in retailing. International Journal of Service Industry Management.

Kim, H. B, y Kim, W. G, (2005). The relationship between brand equity and firms' performance in luxury hotels and chain restaurants. Tourism management, vol. 26, $n^{\circ} 4$, pp. 549-560.

KirkBrown, A, y Wallace, D, (2004). Predicting burnout and job satisfaction in workplace counselors: The influence of role stressors, job challenge, and organizational knowledge. Journal of Employment Counseling, vol. 41, n 1, pp. 29-37.

Kitchen, P.J, y Schultz, D.E, (2009). IMC: New horizon/false dawn for a marketplace in turmoil? Journal of Marketing Communications, vol. 15, n 2-3, pp.197-204.

Kliatchko, J, (2005). Towards a new definition of integrated marketing communications (IMC). International Journal of Advertising, vol. 24, $\mathrm{n}^{\circ}$ 1, pp. 7-34.

Kliatchko, J, (2008). Revisiting the IMC construct - a revised definition and four pillars. International Journal of Advertising, vol. 27, $\mathrm{n}^{\circ}$ 1, pp. 133-160.

Kliatchko, J, (2009). IMC 20 Years after: A Second Look at IMC Definitions. International Journal of Integrated Marketing Communications, vol. 1, $\mathrm{n}^{\mathrm{o}} 2$.

Kliatchko, J. G, (2002). Understanding integrated marketing communications. University of Asia and the Pacific, Institute of Communication.

Kliatchko, J.G, y Schultz, D.E, (2014). Twenty years of imc: A study of ceo and cmo perspectives in the Asiapacific region. International Journal of Advertising, vol. $33, \mathrm{n}^{\circ} 2$, pp. 373.

Low, G. S, (2000). Correlates of integrated marketing communications. Journal of Advertising Research, vol. 40, n 3, pp. 27-39.

Manoli, A. E, y Hodgkinson, I. R, (2020). The implementation of integrated marketing communication (IMC): evidence from professional football clubs in England. Journal of Strategic Marketing, vol. 28, $\mathrm{n}^{\circ}$ 6, pp. 542-563.

McDonald's Corporation Case Study. (2012). McDonalds Case Study: Remaining Relevant in a Health-conscious Society, pp. 1-20.

Mihart, C, (2012). Impact of integrated marketing communication on consumer behaviour: Effects on consumer decision-making process. International Journal of Marketing Studies, vol. 4, n² 2, pp. 121. 
Minor, M. S, Wagner, T, Brewerton, F. J, y Hausman, A, (2004). Rock on! An elementary model of customer satisfaction with musical performances. Journal of services marketing, vol. 18, $\mathrm{n}^{\mathrm{0}}$ 1, pp. 7-18.

Molina, A, Aranda, E, Martín, V. J, y Santos, J, (2014). Opportunities for craft consumption: analysis of the quality perceived by consumers. International Journal of Globalisation and Small Business, vol. 6, $\mathrm{n}^{\mathrm{0}}$ 1, pp. 64-78.

Mora Carpio, J. L, (2013). Uso y utilidad de las comunicaciones integradas al marketing en el mercado de bares restaurantes y discotecas; segmento medio, medio-alto de Cuenca (Bachelor's thesis, Universidad del Azuay).

Moriarty, S, y Schultz, D, (2012). Four theories of how IMC Works. Advertising theory, $\mathrm{n}^{0}$ 3, pp. 491-505.

Mulhern, F, (2013). Integrated marketing communications: From media channels to digital connectivity. The Evolution of Integrated Marketing Communications pp. 19-36. Routledge.

Nakata, C, y Sivakumar, K, (1996). National culture and new product development: An integrative review. The Journal of Marketing, pp. 61-72.

Nowak, G. J, y Phelps, J, (1994). Conceptualizing the integrated marketing communications' phenomenon: An examination of its impact on advertising practices and its implications for advertising research. Journal of Current Issues \& Research in Advertising, vol. 16, n ${ }^{\circ}$ 1, pp. 49-66.

Oguinn, T. C, y Meyer, T. P, (1984). Segmenting the hispanic market-the use of spanishlanguage radio. Journal of Advertising Research, vol. 23, nº 6, pp. 9-16.

Oliver, R. L, (1993). Cognitive, affective, and attribute bases of the satisfaction response. Journal of consumer research, vol. 20, no 3, pp. 418-430.

Oluwafemi, O. J, y Adebiyi, S. O, (2018). Customer Loyalty and Integrated Marketing Communications among Subscribers of Telecommunication Firms in Lagos Metropolis, Nigeria. Journal of Competitiveness, vol. 10, $\mathrm{n}^{\circ}$ 3, pp. 101.

Onkvisit, S, y Shaw, J. J, (2002). Standardized international advertising: some research issues and implications. Journal of Advertising Research, vol. 39, n ${ }^{\circ}$ 6, pp. 19-24.

Ots, M, y Nyilasy, G, (2015). Integrated Marketing Communications (IMC): Why Does It Fail?: An Analysis of Practitioner Mental Models Exposes Barriers of IMC Implementation. Journal of Advertising Research, vol. 55, nº 2, pp. 132-145.

Park, S, Hahn, S, Lee, T, y Jun, M, (2018). Two factor model of consumer satisfaction: International tourism research. Tourism Management, $\mathrm{n}^{\circ}$ 67, pp. 82-88.

Peltier, J. W, Schibrowsky, J. A, y Schultz, D. E, (2003). Interactive integrated marketing communication: combining the power of IMC, the new media and database marketing. International Journal of Advertising, vol. 22, nº 1, pp. 93-115.

Perreault, W. D, (2018). Essentials of marketing. New York: Mc Graw Hill.

Peter, J. P, (1979). Reliability: A review of psychometric basics and recent marketing practices”. Journal of marketing research, $\mathrm{n}^{\circ} 16$, pp. 6-17.

Phillips, D. M, y Baumgartner, H, (2002). The role of consumption emotions in the satisfaction response. Journal of Consumer psychology, vol. 12, n 3 , pp. 243-252.

Porcu, L, (2014). Desarrollo y validación de una escala de medida de la comunicación integrada corporativa: modelización de sus antecedentes y consecuencias en el sector turístico. Granada: Universidad de Granada.

Porcu, L, del Barrio-García, S., Alcántara-Pilar, J.M, y Crespo-Almendros, E, (2016). Do adhocracy and market cultures facilitate firm-wide integrated marketing communication (IMC)? International Journal of Advertising, pp. 1-21. 
Porcu, L, Del Barrio-García, S, y Kitchen, P. J, (2017). Measuring integrated marketing communication by taking a broad organisational approach: The firm-wide IMC scale. European Journal of Marketing, vol. 51, n 3, pp. 692-718.

Premkumar, G, y Bhattacherjee, A, (2008). Explaining information technology usage: A test of competing models. Omega, vol. 36, $\mathrm{n}^{\circ} 1$, pp. 64-75.

Rather, R. A, Tehseen, S, Itoo, M. H, y Parrey, S, H. (2019). Customer brand identification, affective commitment, customer satisfaction, and brand trust as antecedents of customer behavioral intention of loyalty: An empirical study in the hospitality sector. Journal of Global Scholars of Marketing Science, vol. 29, n 2, pp. 196-217.

Rather, R. A, y Sharma, J, (2018). The effects of customer satisfaction and commitment on customer loyalty: Evidence from the hotel industry. Journal of Hospitality Application \& Research, vol. 12, ${ }^{\circ}$ 2, pp. 41-60.

Reid, M, (2005). Performance auditing of integrated marketing communication (IMC) actions and outcomes. Journal of Advertising, vol. 34, n ${ }^{\circ}$ 4, pp. 41-54.

Ryu, H. Y. Kim, H. S, y Shim, J. I, (2009). Rate equation analysis of efficiency droop in InGaN light-emitting diodes. Applied Physics Letters, vol. 95, nº 8, pp. 81-114.

Ryu, K, y Han, H, (2011). New or repeat customers: How does physical environment influence their restaurant experience? International Journal of Hospitality Management, vol. 30, $\mathrm{n}^{\circ}$ 3, pp. 599-611.

Satorra, A, y Bentler, P, (1988). Scaling corrections for statistics in covariance structure analysis.

Schultz, D. E, y Kitchen, P. J, (1997). Integrated marketing communications in US advertising agencies: an exploratory study. Journal of Advertising Research, vol. 37, $\mathrm{n}^{\circ}$ 5, pp. 7-18.

Schultz, D. E, y Kitchen, P. J, (2000). A response to 'Theoretical concept or management fashion. Journal of Advertising Research, vol. 40, no 5, pp. 17-21.

Schultz, D. E, y Schultz, H. F, (1998). Transitioning marketing communication into the twenty-first century. Journal of marketing communications, vol. 4, n 1, pp. 9-26.

Schultz, H, (2003). IMC, The Next Generation: 5 Steps for Delivering Value and Measuring Financial Returns. McGraw-Hill Publishing.

Selvakumar, J. J, (2014). Dimensions of Integrated Marketing Communication (IMC) and Their Impact in Creating Brand Equity in the Quick Service Restaurant (QSR) Industry in Coimbatore City. Ushus-Journal of Business Management, ol. 13, $\mathrm{n}^{\circ}$ 4, pp. 27-40.

Šerić, M, (2018). Content analysis of the empirical research on IMC from 2000 to 2015. Journal of Marketing Communications, vol. 24, $\mathrm{n}^{\circ}$ 7, pp. 647-685.

Šerić, M, Gil-Saura, I, and Ruiz-Molina, M. E, (2014). How can integrated marketing communications and advanced technology influence the creation of customerbased brand equity? Evidence from the hospitality industry. International Journal of Hospitality Management, $\mathrm{n}^{0}$ 39, pp. 144-156.

Sêric, M, y Saura, I. G, (2012). Relaciones causales entre las TIC, la CIM y el valor de marca: un estudio a partir de las percepciones de los huéspedes. Papers de Turisme, $\mathrm{n}^{\circ}$ 52, pp. 1-21.

Sheehan, M, (2006). McDonald's in crisis: A comparative analysis in a national organizational context. Competition Forum, vol. 4, no 1, pp. 221-227.

Singh, S, (2006). Cultural differences in, and influences on, consumers' propensity to adopt innovations. International Marketing Review, vol. 23, n 2, pp. 173-191.

Tafesse, W, y Kitchen, P. J, (2017). IMC-an integrative review. International Journal of Advertising, vol. 36, $\mathrm{n}^{\mathrm{o}}$ 2, pp. 210-226. 
Tjiptono, F, y Diana, A, (2003). Total Quality Management, Yogyakarta. Andi Offset.

Torp, S, (2009). Integrated communications: from one look to normative consistency. Corporate Communications: An International Journal, vol. 14, n 2, pp. 190-206.

Vignali, C, (2001). McDonald's: “think global, act local”-the marketing mix. British Food Journal, vol. 103, n 2, pp. 97-111.

Vorhies, D. W, Morgan, R. E, y Autry, C. W, (2009). Product-market strategy and the marketing capabilities of the firm: impact on market effectiveness and cash flow performance. Strategic Management Journal, vol. 30, n 12, pp. 1310-1334.

Welch, D, y Tierney, C, (2000). "Can the Mondeo Get Ford Back into the Race? Business Week, $\mathrm{n}^{0} 3703$, pp. 74-74.

Wilson, A, (2002). Attitudes towards customer satisfaction measurement in the retail sector. International Journal of Market Research, vol. 44, n 2, pp. 213.

Wu, C. H. J, y Liang, R. D, (2009). Effect of experiential value on customer satisfaction with service encounters in luxury-hotel restaurants. International Journal of Hospitality Management, vol. 28, nº 4, pp. 586-593. 